\title{
Nonthermal Nuclei in 374 UGC Galaxies
}

\author{
J. M. Wrobel
}

National Radio Astronomy Observatory, Socorro, NM, U.S.A.

\section{J. J. Condon}

National Radio Astronomy Observatory, Charlottesville, VA, U.S.A.

\section{J. Machalski}

\author{
Astronomical Observatory, Jagellonian University, Cracow, Poland
}

Abstract. The VLA was used at $1.5-2$ arcsec resolution at $20 \mathrm{~cm}$ to search for compact continuum sources in the inner regions of 374 UGC galaxies.

Black holes in galactic nuclei can leave photometric evidence for their presence. A robust photometric tracer of these "monsters" is synchrotron emission from a nonthermal radio nucleus. At 1 arcsec resolution, such a nucleus would appear as a compact radio source in a galaxy's inner regions. These sources are useful as astrometric tracers of black holes, and offer proof of current nuclear activity, even when most galactic radio emission originates hundreds of kiloparsecs from the nucleus. Furthermore, absorption line studies toward compact radio sources can constrain models for fueling black holes and models for tori obscuring black holes.

Surveys for compact radio sources should target UGC galaxies (Nilson 1973) because of their low ratio of linear to angular scale. UGC galaxies are also near enough for good Hubble type assignments; curiously, these beaming-independent types correlate with radio properties, offering a handle on unified schemes unavailable for samples of distant, radio-selected galaxies (Condon, Frayer, \& Broderick 1991). In addition, a few UGC galaxies are well studied at radio wavelengths, and provide compelling evidence for relativistic jets on parsec scales which appear to decelerate to subrelativistic speeds on kiloparsec scales (Bicknell 1994); more examples are needed.

Condon \& Broderick (1988) and Condon, Frayer, \& Broderick (1991) defined two samples of 374 UGC galaxies, selected independent of Hubble type from Green Bank sky maps at $20 \mathrm{~cm}$ and $6 \mathrm{~cm}$, to limiting total flux densities of $150 \mathrm{mJy}$ and $25 \mathrm{mJy}$, respectively. They used infrared-radio flux ratios, infrared spectral indices, and radio morphologies to classify the total radio energy source for each galaxy as a "monster" or a "starburst". Typical distances to "monster" and "starburst" galaxies are $130 \mathrm{Mpc}$ and $30 \mathrm{Mpc}$, respectively, based on distances from Sandage \& Tammann (1981) or assuming a Hubble flow with $H_{0}=50 \mathrm{~km} \mathrm{~s}^{-1} \mathrm{Mpc}^{-1}$.

We used the VLA at $20 \mathrm{~cm}$ to image these two UGC galaxy samples with a resolution of 1.5-2 arcsec and a typical rms sensitivity of $0.2 \mathrm{mJy}^{\text {beam }}{ }^{-1}$. The radio peak was found for the inner 40 by $40 \operatorname{arcsec}^{2}$ region of each galaxy. The peak VLA power is plotted as a function of galaxy distance in Fig. 1. Peaks referring to detections of compact sources are plotted as circles. If only extended emission was detected, then the peak power is plotted as a square. Plot symbols further distinguish the total radio energy source as "monster" or "starburst".

Compact VLA sources stronger than $1 \mathrm{mJy}$ are detected in $90 \%$ of "monster" galaxies and $20 \%$ of "starburst" galaxies. A few of these compact sources 


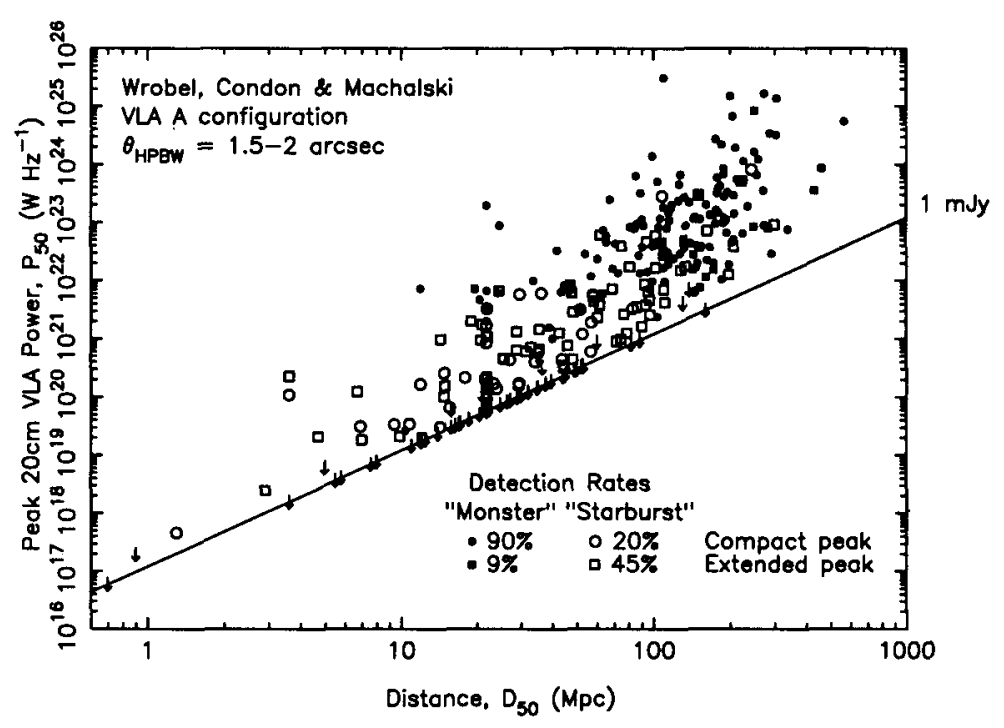

Figure 1. Dependence of peak VLA power on distance for 374 UGC galaxies selected from Green Bank sky surveys. Confusion will cause about four false detections.

were known from past work (e.g., Condon \& Dressel 1978; Condon et al. 1982, 1991; Wrobel \& Heeschen 1984, 1988, 1991) but the vast majority represent new discoveries. At a typical distance the host galaxies of the "monsters" are usually ellipticals or lenticulars, and the linear resolution is $0.9-1.3 \mathrm{kpc}$. At a typical distance the host galaxies of the "starbursts" are usually spirals or unclassified, and the linear resolution is 220-290 pc. These compact VLA sources should be filtered for high brightness temperatures with the VLBA, to determine which are bona fide nonthermal nuclei able to serve as as photometric and astrometric tracers of black holes in UGC galaxies.

Acknowledgments. The National Radio Astronomy Observatory is a facility of the National Science Foundation, operated under a cooperative agreement by Associated Universities, Inc.

\section{References}

Bicknell, G. V. 1994. ApJ, 422, 542-561.

Condon, J. J., \& Dressel, L. L. 1978. ApJ, 221, 456-467.

Condon, J. J., \& Broderick, J. J. 1988. $A J, 96,30-61$.

Condon, J. J., Frayer, D. T., \& Broderick, J. J. 1991. AJ, 101, 362-409.

Condon, J. J., et al. 1982. ApJ, 252, 102-124.

Condon, J. J., et al. 1991. $A p J, 378,65-76$.

Nilson, P. 1973. Uppsala General Catalog of Galaxies (Uppsala: Uppsala Astronomical Observatory).

Sandage, A., \& Tammann, G. A. 1981. A Revsied Shapley-Ames Catalog of Bright Galaxies (Washington: Carnegie Institution of Washington).

Wrobel, J. M., \& Heeschen, D. S. 1984. ApJ, 287, 41-65.

Wrobel, J. M., \& Heeschen, D. S. 1988. $A p J, 335,677-687$.

Wrobel, J. M., \& Heeschen, D. S. 1991. $A J, 101,148-169$. 